\title{
Solvent Free One-Pot Synthesis of 1,2,4,5-Tetrasubstituted Imidazoles Catalyzed by Secondary Amine Based Ionic Liquid and Defective Keggin Heteropoly Acid
}

\author{
Pranab Jyoti Das ${ }^{1 *}$, Jupitara Das ${ }^{1}$, Munni Ghosh ${ }^{1}$, Sabera Sultana ${ }^{2}$ \\ ${ }^{1}$ Department of Chemistry, Gauhati University, Guwahati, India \\ ${ }^{2}$ Indian Institute of Technology, Guwahati, India \\ Email: *pjd123@sify.com
}

Received August 31, 2013; revised September 30, 2013; accepted October 7, 2013

Copyright (C) 2013 Pranab Jyoti Das et al. This is an open access article distributed under the Creative Commons Attribution License, which permits unrestricted use, distribution, and reproduction in any medium, provided the original work is properly cited.

\begin{abstract}
Secondary amine based ionic liquid and defective Keggin type heteropoly acid (HPA) are separately used for efficient one-pot four-component synthesis of 1,2,4,5-tetrasubstituted imidazoles assisted by microwave (MW). Eco-friendly solvent free procedure, short reaction time, high yield of products and reusability of catalysts are important features of the synthesis. A comparative study on the efficiency of the two catalysts is reported. This work further demonstrates the alternate use of urea, instead of often used ammonium acetate, as source of nitrogen.
\end{abstract}

Keywords: Ionic Liquids; Keggin Heteropolyacid; Microwave; Tetrasubstituted Imidazole; Solvent Free Synthesis; Multicomponent Reaction

\section{Introduction}

Tetrasubstituted imidazoles are important heterocycles with wide biological applications. Their synthesis and reactions form a significant part of the study of heterocycles with medicinal use [1]. Tetrasubstituted imidazole scaffold is the most active constituent in many biological systems and drug molecules such as olmesartan medoxomil, losartan, eprosartan and trifenagrel [2-4] as well as other natural products of pharmaceutical importance [5-7]. Multicomponent reaction (MCR) is a powerful tool in generating molecules with diverse functionality in a single synthetic protocol [8-10] and forms the basis of the synthesis of 1,2,4,5-tetrasubstituted imidazoles. Important synthetic methods include one-pot thiazolium catalyzed addition of an aldehyde to an acyl imine followed by ring closure to the imidazole [11], condensation of arylglyoxal, $1^{\circ}$-amine, carboxylic acids and isocyanates on Wang resin followed by cyclization in the presence of acetic acid [12], by a hetero-Cope rearrangement [13], a direct synthesis from alkenes involving two step ketoiodonation/cyclization protocol [14], Domino reaction of 2-azido acrylates and nitrones [15].

"Corresponding author.
Other synthetic methods include the condensation of benzoin and benzoin acetate with aldehyde, $1^{\circ}$-amine, ammonia in the presence of copper acetate [16], one-pot synthesis using $\mathrm{BF}_{3}-\mathrm{SiO}_{2}$ as catalyst [17], cyclization of sulphonamide with mesoionic 1,3-oxazolinium-5-olates [18], condensation of $\beta$-carbonyl-N-acyl-N-alkylamines with $\mathrm{NH}_{4} \mathrm{OAc}$ in refluxing acetic acid $[19,20]$, conversion of N-2-oxoamides with ammonium triflate [21] besides others [22-25]. Several catalysts have been explored for performing the four components' reaction to give the target product notably the use of L-proline [26], zeolite HY and silica gel [27], Keggin type heteropolyacid [28], $\mathrm{LaCl}_{3}$ catalyzed synthesis using urea as a source of ammonia [29]. Shaterian et al. reported the synthesis of tetrasubstituted imidazole used Brønsted acidic ionic liquid, N-methyl-2-pyrrolidonium hydrogen sulphate, as catalyst, but long reaction time, high amount of catalyst used and the time-consuming procedure for the preparation of the IL (12 hrs) are some of the disadvantages [30]. Most of the procedures reported are associated with one or more disadvantages such as use of expensive reagents and catalysts, mineral acids and requirement of large amount of catalysts which eventually results in generation of toxic wastes, longer reaction time and tedious 
work-up.

\section{Results and Discussion}

In a broad programme of developing efficient, selective and eco friendly synthetic methods for pharmacologically important heterocycles, we explored the applicability of green promoters namely the simple acidic ionic liquids (IL) as well as the defective Keggin type heteropolyacids (HPA) in the synthesis of 1,2,4,5 tetrasubstituted imidazoles using microwave (MW) heating in both procedures. Combination of two green techniques is reported to give better results in terms of short reaction time and high yield of products. Catalytic efficiencies of both the ionic liquid and heteropolyacid are well documented. The unique thermodynamic properties of both are increasingly enticing chemists to explore their use as media as well as promoters in organic synthesis [31-33], however, high cost of the often used imidazole based ILs and the heteropoly acids deter their use in industrial processes. Herein, we explored the possibility of using simple and cost effective secondary amine based IL namely di- $n$-propylammonium hydrogensulphate in the synthesis of imidazoles in a solvent free multicomponent reaction (MCR) using $\mathrm{MW}$ heating. In a related study, the efficiency of this IL vis a vis a heteroploy acid as an alternative catalyst for the synthesis was examined under identical experimental conditions. Initially the IL and the HPA used were prepared by adopting a simple atom economy procedure using cheap and easily available substrates. The IL used herein was prepared by the action of conc. $\mathrm{H}_{2} \mathrm{SO}_{4}$ on di- $n$-propylamine [34] and the lacunary and defective Keggin type HPA namely $\mathrm{H}_{6} \mathrm{PAlMo}_{11} \mathrm{O}_{40}$ was prepared by a reported procedure [35] albeit with minor modification. Microwave heating was used while preparing the HPA instead of conventional heating which resulted in less reaction time and high yield of the HPA.

For the purpose of optimization of the amount of catalyst and the reaction time, representative reaction using benzil, benzaldehyde, benzylamine and $\mathrm{NH}_{4} \mathrm{OAc}$ as the substrates was performed by varying the concentration of the catalyst from $5 \mathrm{~mol} \%$ to $10 \mathrm{~mol} \%$ and concomitant variation of reaction time from 2 mins to 10 mins. In case of IL as the catalyst, yield of the desired product $\mathbf{4 a}$ improved by $20 \%$ when mol $\%$ was increased from $5 \%$ to $10 \%$ and in case of HPA $5 \mathrm{~mol} \%$ of catalyst and $10 \mathrm{~min}$ reaction time gave the best results. No improvement in yield was apparent with increase in $\mathrm{mol} \%$ of catalyst and/or the reaction time. Results summarized in Table $\mathbf{1}$ indicated best results with $10 \mathrm{~mol} \%$ of IL as catalysts and in case of HPA it was $5 \mathrm{~mol} \%$.

These two catalysts were used in turn for performing a one-pot four component reaction involving 1,2-diketone, aromatic aldehyde, $1^{\circ}$-amine and $\mathrm{NH}_{4} \mathrm{OAc}$ to give the target 1,2,4,5-tetrasubstituted imidazole under solvent free condition mediated by MW. In a typical procedure, the reactants and the catalyst in the form of either HPA or IL, are thoroughly mixed and exposed to MW irradiation. In order to establish the generality of the synthetic procedure, several diversified examples were studied by varying the $1^{\circ}$-amine and the aldehyde. The yields obtained by using both HPA and the IL are good to excellent with very little or no side products which is a significant improved over other reported procedures. Further good results have been obtained with both the aliphatic as well as the aromatic amines. Reactions with the IL as promoter required 2 - 3 min for completion and reactions catalyzed by HPA required $10-13 \mathrm{~min}$. These results are indicative of better efficiency of IL over HPA in this synthesis. The reaction is shown in Scheme $\mathbf{1}$ and the results summarized in Table 2. Work up was accomplished by extraction of the reaction mixture with $\mathrm{CH}_{2} \mathrm{Cl}_{2}$ to separate out the target product. The $\mathrm{CH}_{2} \mathrm{Cl}_{2}$ solution was washed with water $(3 \times 50 \mathrm{~mL})$ to remove any trace of the impurity. Since both the catalysts are insoluble in $\mathrm{CH}_{2} \mathrm{Cl}_{2}$ the catalysts were precipitated out after addition of $\mathrm{CH}_{2} \mathrm{Cl}_{2}$ to the reaction mixture and recovered by simple filtration. The recovered IL was stored in desiccator for reuse and HPA was dried at $85^{\circ} \mathrm{C}$ for 24 hours and reused. Both the catalysts retained their activity after two subsequent reuses.

In order to preclude other possibilities, the reaction was examined using related catalyst namely tetra- $n$ propylammonium bromide and $\mathrm{NaHSO}_{4}$ under MW irradiation. With tetra- $n$-propylammonium bromide, the reaction did not proceed at all whereas with $\mathrm{NaHSO}_{4}$ about

Table 1. Effect of amount of catalyst for the formation of $4 a$ via the 4-MCR of 1, 2, 3 and $\mathrm{NH}_{4} \mathrm{OAc}$.

\begin{tabular}{cccccc}
\hline \multirow{2}{*}{ Entry } & \multirow{2}{*}{ mol\% } & \multicolumn{2}{c}{ Ionic liquid } & \multicolumn{2}{c}{ Heteropoly acid } \\
\cline { 3 - 6 } & & Time (min) & Yield $(\%)^{\mathrm{a}}$ & Time (min) & Yield (\%) \\
\hline 1 & 5 & 2 & 65 & 2 & trace \\
2 & 5 & 10 & 69 & 10 & 89 \\
3 & 10 & 2 & 85 & 2 & trace \\
4 & 10 & 10 & 86 & 10 & 89 \\
\hline
\end{tabular}

a. Yields refer to the pure isolated product.

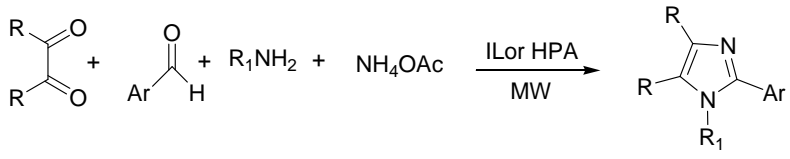

$$
\begin{aligned}
& 1234 \text { (a-s) } \\
& \mathrm{R}=\text { Phenyl, p-tolyl; } \\
& \mathrm{R} 1=\mathrm{C}_{6} \mathrm{H}_{5^{-}}, \mathrm{C}_{6} \mathrm{H}_{5} \mathrm{CH}_{2^{-}}, 4-\mathrm{NO}_{2} \mathrm{C}_{6} \mathrm{H}_{4^{-}}, \mathrm{C}_{2} \mathrm{H}_{5^{-}}, \mathrm{CH}_{3}- \\
& \mathrm{IL}=\left[\mathrm{n}-\mathrm{Pr}_{2} \mathrm{NH}_{2}\right]\left[\mathrm{HSO}_{4}\right], \mathrm{HPA}=\mathrm{H}_{6} \mathrm{PAlMo}_{11} \mathrm{O}_{40}
\end{aligned}
$$

Scheme 1. Synthesis of imidazole derivatives mediated by IL/HPA. 
Table 2. Solvent free MW induced one-pot synthesis of 1, 2, 4, 5-tetrasubstituted imidazole promoted by $\left[n-\mathrm{Pr}_{2} \mathrm{NH}_{2}\right]\left[\mathrm{HSO} \mathrm{H}_{4}\right]$ or HPA using ammonium acetate as nitrogen source.

\begin{tabular}{|c|c|c|c|c|c|c|c|}
\hline \multirow{2}{*}{ Product } & \multirow{2}{*}{$\mathrm{R}$} & \multirow{2}{*}{$\mathrm{Ar}$} & \multirow{2}{*}{$\mathrm{R}_{1}$} & \multicolumn{2}{|c|}{ Reaction with IL } & \multicolumn{2}{|c|}{ Reaction with HPA } \\
\hline & & & & Time (min) & Yield $(\%)^{\mathrm{a}}$ & Time (min) & Yield $(\%)^{\mathrm{a}}$ \\
\hline $4 a$ & $\mathrm{Ph}$ & $\mathrm{C}_{6} \mathrm{H}_{5}$ & $\mathrm{C}_{6} \mathrm{H}_{5} \mathrm{CH}_{2}$ & 2 & 85 & 10 & 89 \\
\hline $4 b$ & $\mathrm{Ph}$ & 4- $\mathrm{CH}_{3} \mathrm{C}_{6} \mathrm{H}_{4}$ & $\mathrm{C}_{6} \mathrm{H}_{5} \mathrm{CH}_{2}$ & 2 & 87 & 10 & 92 \\
\hline $4 c$ & $\mathrm{Ph}$ & 2- $\mathrm{CH}_{3} \mathrm{OC}_{6} \mathrm{H}_{4}$ & $\mathrm{C}_{6} \mathrm{H}_{5} \mathrm{CH}_{2}$ & 2 & 87 & 10 & 94 \\
\hline $4 d$ & $\mathrm{Ph}$ & $3,4-\left(\mathrm{OCH}_{3}\right)_{2} \mathrm{C}_{6} \mathrm{H}_{3}$ & $\mathrm{C}_{6} \mathrm{H}_{5} \mathrm{CH}_{2}$ & 2 & 87 & 10 & 93 \\
\hline $4 e$ & $\mathrm{Ph}$ & $4-\mathrm{ClC}_{6} \mathrm{H}_{4}$ & $\mathrm{C}_{6} \mathrm{H}_{5} \mathrm{CH}_{2}$ & 2 & 85 & 13 & 90 \\
\hline $4 f^{b}$ & $\mathrm{Ph}$ & 4- $\mathrm{EtC}_{6} \mathrm{H}_{4}$ & $\mathrm{C}_{6} \mathrm{H}_{5} \mathrm{CH}_{2}$ & 2 & 87 & 10 & 92 \\
\hline $4 g$ & $\mathrm{Ph}$ & $3-\mathrm{BrC}_{6} \mathrm{H}_{4}$ & $\mathrm{C}_{6} \mathrm{H}_{5} \mathrm{CH}_{2}$ & 2 & 85 & 10 & 89 \\
\hline $4 h$ & $\mathrm{Ph}$ & $4-\mathrm{NO}_{2} \mathrm{C}_{6} \mathrm{H}_{4}$ & $\mathrm{C}_{6} \mathrm{H}_{5} \mathrm{CH}_{2}$ & 2 & 85 & 10 & 88 \\
\hline $4 i^{\mathrm{b}}$ & $\mathrm{Ph}$ & $2,5-\left(\mathrm{CH}_{3}\right)_{2} \mathrm{C}_{6} \mathrm{H}_{4}$ & $\mathrm{C}_{6} \mathrm{H}_{5}$ & 2 & 84 & 10 & 90 \\
\hline $4 j$ & $\mathrm{Ph}$ & $\mathrm{C}_{6} \mathrm{H}_{5}$ & $\mathrm{C}_{6} \mathrm{H}_{5}$ & 2 & 83 & 10 & 87 \\
\hline $4 k^{b}$ & $\mathrm{Ph}$ & 4- $\mathrm{ClC}_{6} \mathrm{H}_{4}$ & $\mathrm{CH}_{3}$ & 3 & 87 & 10 & 89 \\
\hline $41^{\mathrm{b}}$ & $\mathrm{Ph}$ & 4- $\mathrm{EtC}_{6} \mathrm{H}_{4}$ & $\mathrm{CH}_{3}$ & 3 & 86 & 10 & 89 \\
\hline $4 m^{b}$ & $\mathrm{Ph}$ & 2- $\mathrm{CH}_{3} \mathrm{OC}_{6} \mathrm{H}_{4}$ & $\mathrm{CH}_{3} \mathrm{CH}_{2}$ & 3 & 90 & 10 & 92 \\
\hline $4 n$ & $\mathrm{Ph}$ & $4-\mathrm{ClC}_{6} \mathrm{H}_{4}$ & $\mathrm{CH}_{3} \mathrm{CH}_{2}$ & 3 & 90 & 10 & 92 \\
\hline $40^{b}$ & $\mathrm{Ph}$ & $3,4-\left(\mathrm{OCH}_{3}\right)_{2} \mathrm{C}_{6} \mathrm{H}_{3}$ & $\mathrm{CH}_{3} \mathrm{CH}_{2}$ & 3 & 90 & 10 & 92 \\
\hline $4 p^{b}$ & $4-\mathrm{CH}_{3} \mathrm{Ph}$ & $2,6-\mathrm{Cl}_{2} \mathrm{C}_{6} \mathrm{H}_{3}$ & $\mathrm{CH}_{3} \mathrm{CH}_{2}$ & 3 & 92 & 10 & 92 \\
\hline $4 q^{b}$ & $\mathrm{Ph}$ & 4- $\mathrm{ClC}_{6} \mathrm{H}_{4}$ & $4-\mathrm{NO}_{2} \mathrm{C}_{6} \mathrm{H}_{4}$ & 3 & 85 & 13 & 87 \\
\hline $4 r^{b}$ & $\mathrm{Ph}$ & $3,4-\left(\mathrm{OCH}_{3}\right)_{2} \mathrm{C}_{6} \mathrm{H}_{3}$ & $4-\mathrm{NO}_{2} \mathrm{C}_{6} \mathrm{H}_{4}$ & 3 & 85 & 13 & 88 \\
\hline $4 s$ & $\mathrm{Ph}$ & $\mathrm{C}_{6} \mathrm{H}_{5}$ & $4-\mathrm{NO}_{2} \mathrm{C}_{6} \mathrm{H}_{4}$ & 3 & 83 & 13 & 85 \\
\hline
\end{tabular}

a. Yields refers to the pure isolated products; $b$. New compound.

$40 \%$ yield of desired product was obtained indicating the essentiality of di- $n$-propylammonium hydrogensulphate as the appropriate catalyst for the reaction. The results obtained with HPA, $\left[n-\mathrm{Pr}_{2} \mathrm{NH}_{2}\right]\left[\mathrm{HSO}_{4}\right], \mathrm{NaHSO}_{4}, n$ $\mathrm{Pr}_{4} \mathrm{NBr}$ and in absence of catalyst for a model reaction are summarized in Table 3. In this study benzil, 2-anisaldehyde, benzyl amine and ammonium acetate were taken as the reference reactants and $10 \mathrm{~mol} \%$ amounts of catalysts were used.

Investigations were also carried out using urea as the source of nitrogen in the reaction. It was observed that the reaction proceeded equally well with urea in the presence of both IL and HPA. Here efficiency was examined in some model reactions in presence of either IL or HPA. The results obtained by using urea instead of $\mathrm{NH}_{4} \mathrm{OAc}$ are summarized in Table 4.

All products obtained were characterized by spectroscopic method such as IR, ${ }^{1} \mathrm{H}$ NMR, ${ }^{13} \mathrm{C}$ NMR, Mass spectrometry and by comparing their melting points with those reported in literature. Some new compounds are also reported. Single crystal X-ray analysis of one of the new products namely 1-ethyl-2-(2', 6'-dichlorophenyl)-4, 5-di(4'-methylphenyl)-imidazole (4p) confirms the structure (Figure 1). Crystals were obtained from ethanol in which case the results indicate the presence of residual
Table 3. Effect of catalyst on the one-pot four component synthesis of tetra substituted imidazole $4 c$ with the usual reactants and $\mathrm{NH}_{4} \mathrm{OAC}$.

\begin{tabular}{cccc}
\hline Entry & Catalyst $^{\mathrm{b}}$ & Time (min) & Yield (\%) $^{\mathrm{a}}$ \\
\hline 1 & Catalyst free & 10 & - \\
2 & $\mathrm{HPA}$ & 10 & 95 \\
3 & {$\left[n-\mathrm{Pr}_{2} \mathrm{NH}_{2}\right]\left[\mathrm{HSO}_{4}\right]$} & 10 & 88 \\
4 & $\mathrm{NaHSO}_{4}$ & 10 & 40 \\
5 & $n-\mathrm{Pr}_{4} \mathrm{NBr}$ & 10 & - \\
\hline
\end{tabular}

a. Yields refer to the pure isolated product; b. $10 \mathrm{~mol} \%$ amount of catalysts were used.

solvent in the crystal. Attempt to remove the solvent destroyed crystallinity of the product and no worthwhile data could be obtained. Crystallographic data are given in Table 5. CCDC reference number for the compound is CCDC 892443.

\section{Conclusion}

In conclusion, a one-pot solvent free MCR promoted by the combined use of microwave and IL or HPA offer easy access to tetrasubstituted imidazoles in excellent yield. Procedural simplicity, short reaction time, solvent 
Table 4. The effect of ammonium acetate and urea as the nitrogen source on the 4-MCR to form $4 \mathrm{a}, 4 \mathrm{~d}$ and $4 \mathrm{~m}$.

\begin{tabular}{ccccccc}
\hline \multirow{2}{*}{ Entry } & \multirow{2}{*}{ Nitrogen source } & Product & \multicolumn{3}{c}{ IL } & \multicolumn{2}{c}{ HPA } \\
\cline { 4 - 7 } & & Time (min) & Yield (\%) $^{\mathrm{a}}$ & Time (min) & Yield (\%) $^{\mathrm{a}}$ \\
\hline 1 & Ammonium acetate & $\mathbf{4 a}$ & 2 & 86 & 10 & 89 \\
2 & Ammonium acetate & $\mathbf{4 d}$ & 2 & 86 & 10 & 95 \\
3 & Ammonium acetate & $\mathbf{4 m}$ & 3 & 90 & 10 & 90 \\
4 & Urea & $\mathbf{4 a}$ & 2 & 86 & 10 & 89 \\
5 & Urea & $\mathbf{4 d}$ & 2 & 86 & 10 & 95 \\
6 & Urea & $\mathbf{4 m}$ & 3 & 90 & 10 & 90 \\
\hline
\end{tabular}

a. Yields refer to the pure isolated products.

Table 5. Crystallographic parameter of compound 4p.

\begin{tabular}{cc}
\hline empirical formula & $\mathrm{C}_{25} \mathrm{H}_{22} \mathrm{Cl}_{2} \mathrm{~N}_{2} \cdot \mathrm{C}_{2} \mathrm{H}_{5} \mathrm{OH}$ \\
formula weight & 467.43 \\
crystal system & Monoclinic \\
space group & $P 2(1) / \mathrm{n}$ \\
$\mathrm{T}(\mathrm{K})$ & 298 \\
$a / \AA$ & $11.7711(9)$ \\
$b / \AA$ & $15.5380(12)$ \\
$c / \AA$ & $13.9410(12)$ \\
$\alpha /$ deg & 90.00 \\
$\beta /$ deg & $95.289(5)$ \\
$\gamma /$ deg & 90.00 \\
$\mathrm{~V} / \AA^{3}$ & $2538.9(4)$ \\
$\mathrm{D}_{\text {calcd }}\left(\mathrm{g} \cdot \mathrm{cm}{ }^{-3}\right)$ & 1.223 \\
$\mu\left(\mathrm{mm}{ }^{-1}\right)$ & 0.271 \\
$\mathrm{Z}$ & 4 \\
reflns collected & 25872 \\
unique reflns & 7416 \\
Observed reflns & 3022 \\
$\mathrm{R}_{1}[\mathrm{I}>2 \sigma(\mathrm{I})]$ & 0.0837 \\
$w \mathrm{R} 2($ all $)$ & 0.1546 \\
goodness-of-fit & 1.287 \\
diffractometer & $\mathrm{SMART} \mathrm{Bruker}$ Apex-II \\
\hline
\end{tabular}

free condition and the use of non toxic and environmentally benign promoters are some of the important features of this new methodology. An added advantage is the simple recovery and efficient reusability of the catalyst. The reaction proceeds equally well with urea as a source of nitrogen instead of $\mathrm{NH}_{4} \mathrm{OAc}$ which result in cost reduction of the transformation.

\section{Experimental Section}

\subsection{General}

Melting points were recorded in a VMP-D model melting point apparatus and are uncorrected. ${ }^{1} \mathrm{H}$ NMR and ${ }^{13} \mathrm{C}$ NMR spectra were recorded on a Bruker advance digital $300 \mathrm{MHz}$ spectrometer in $\mathrm{CDCl}_{3}$. TMS was used as in-

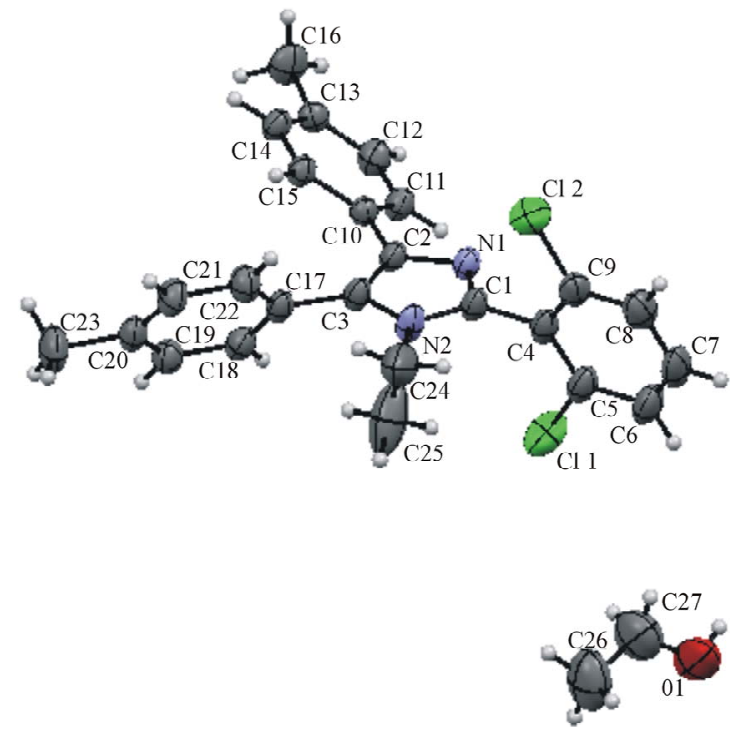

Figure 1. ORTEP diagram of $4 p$.

ternal standard. IR spectra were obtained on a Perkin Elmer FT-IR 1600 spectrophotometer using $\mathrm{KBr}$ pallets. Mass spectra were recorded on a Waters Micromass $\mathrm{ZQ}^{\mathrm{TM}} 400$ Mass spectrometer system. The XRF analysis was carried out in Axios XRF Spectrometer. Microwave irradiation of the reaction mixture was performed in a reactor procured from Catalyst ${ }^{\mathrm{TM}}$ (India) reactor. The single crystal X-ray diffraction data for compound 1ethyl-2-(2', 6'-dichlorophenyl)-4, 5-di(4'-methylphenyl) imidazole (4p) was collected on a SMART Bruker ApexII diffractometer. The structure was solved by direct method and refined by full-matrix least squares based on $F^{2}$ using the SHELXTL 5.1 software package. The hydrogen atoms residing in the carbon atoms were located geometrically. All non-hydrogen atoms were refined anisotropically.

\subsection{Synthetic Procedure for the Preparation of the $\mathrm{H}_{6} \mathrm{PAlMo}_{11} \mathrm{O}_{40}$}

A stoichiometric mixture of $\mathrm{H}_{3} \mathrm{PO}_{4} 85 \%(0.58 \mathrm{~g}, 0.01$ $\mathrm{mol}), \mathrm{Al}_{2} \mathrm{O}_{3} \cdot 6 \mathrm{H}_{2} \mathrm{O}(1.21 \mathrm{~g}, 0.005 \mathrm{~mol})$ and $\mathrm{MoO}_{3}(14.4 \mathrm{~g}$, 
$0.10 \mathrm{~mol})$ was suspended in $150 \mathrm{ml}$ of distilled water and irradiated with microwave for 10 minutes $(560 \mathrm{~W})$. On cooling the mixture to room temperature the insoluble $\mathrm{MoO}_{3}$ precipitated and recovered by filtered. Reduced pressure removal of water and drying at $85^{\circ} \mathrm{C}$ for 24 hours gave dark green crystals of composition $\mathrm{H}_{6} \mathrm{PAlMo}_{11} \mathrm{O}_{40}$ identical to that obtained earlier [35]. The metal composition was established by XRF analysis. IR (KBR): $v$ 1074, 982, 875, 765, 362 and $345 \mathrm{~cm}^{-1}$.

\subsection{General Procedure for the Synthesis of Tetrasubstituted Imidazole}

A mixture of 1, 2-diketone (1 mmol), aldehydes (1 $\mathrm{mmol})$, primary amines (1 mmol), ammonium acetate (or urea) $(1.5 \mathrm{mmol})$ and IL (10 mol\%)/HPA (5 mol\%) was irradiated with microwave $(560 \mathrm{~W})$ with stirring for 1 3/10 - 13 min as mentioned in Table 2. On completion of the reaction monitored by TLC, the reaction mixture was extracted with $\mathrm{CH}_{2} \mathrm{Cl}_{2}(5 \mathrm{~mL} \times 3)$, washed with water, dried with anhydrous $\mathrm{Na}_{2} \mathrm{SO}_{4}$ and solvent removed under reduced pressure. The crude products were purified by recrystallization from ethanol.

1-Benzyl-2,4,5-triphenylimidazole (4a): Mp 159 $161^{\circ} \mathrm{C}(\mathrm{EtOH}) ; \mathrm{IR}(\mathrm{KBr}): v 2890(\mathrm{CH}), 1590(\mathrm{CN}), 1480$ $\mathrm{cm}^{-1} ;{ }^{1} \mathrm{H}$ NMR $\left(300 \mathrm{MHz}, \mathrm{CDCl}_{3}\right): \delta_{\mathrm{H}} \mathrm{ppm} 7.58-6.82$ $(\mathrm{m}, 20 \mathrm{H}, \mathrm{Ar}), 5.12\left(\mathrm{~s}, 2 \mathrm{H}, \mathrm{CH}_{2}\right) ;{ }^{13} \mathrm{C} \mathrm{NMR}(75 \mathrm{MHz}$, $\left.\mathrm{CDCl}_{3}\right): \delta$ ppm 148.08, 138.07, 137.56, 134.46, 131.08, 131.02 , 130.94, 130.05, 129.08, 128.92, 128.81, 128.61, $128.59,128.09,127.36,126.78,126.37,126.02,48.28$.

1-Benzyl-2-(4'-methylphenyl)-4,5-diphenylimidazole (4b): Mp $164-166^{\circ} \mathrm{C}$ (EtOH); IR(KBr): v $2885(\mathrm{CH})$, $1580(\mathrm{CN}), 1482 \mathrm{~cm}^{-1} ;{ }^{1} \mathrm{H}$ NMR $\left(300 \mathrm{MHz}, \mathrm{CDCl}_{3}\right): \delta_{\mathrm{H}}$ ppm 7.58 - 6.81 (m, 19H, Ar), 5.11 (s, 2H, $\left.\mathrm{CH}_{2}\right), 2.38$ (s, $\left.3 \mathrm{H}, \mathrm{CH}_{3}\right) ;{ }^{13} \mathrm{C}$ NMR (75 MHz, $\left.\mathrm{CDCl}_{3}\right): \delta$ ppm 148.21, 138.84, 137.67, 134.52, 131.07, 129.28, 128.94, 128.75, 128.55, 128.06, 126.78, 125.99, 48.24, 21.36.

1-Benzyl-2-(2'-methoxyphenyl)-4,5-diphenylimidazole (4c): Mp $188-190^{\circ} \mathrm{C}(\mathrm{EtOH})$; IR(KBr): v $2823(\mathrm{CH})$, 1593 (CN), 1460 (CC), $1250 \mathrm{~cm}^{-1}$; ${ }^{1} \mathrm{H}$ NMR (300 MHz, $\left.\mathrm{CDCl}_{3}\right): \delta_{\mathrm{H}}$ ppm $7.59-6.82(\mathrm{~m}, 19 \mathrm{H}, \mathrm{Ar}), 5.09(\mathrm{~s}, 2 \mathrm{H}$, $\left.\mathrm{CH}_{2}\right), 3.83\left(\mathrm{~s}, 3 \mathrm{H}, \mathrm{OCH}_{3}\right) ;{ }^{13} \mathrm{C} \mathrm{NMR}\left(75 \mathrm{MHz}, \mathrm{CDCl}_{3}\right): \delta$ ppm 160.04, 147.94, 137.61, 131.02, 130.37, 128.72, $128.53,128.02,127.27,126.72,126.25,125.92,123.29$, 113.95, 55.27, 48.16; Anal. Calcd for $\mathrm{C}_{29} \mathrm{H}_{24} \mathrm{~N}_{2} \mathrm{O}$ : C, 83.63; H, 5.81; N, 6.73, O, 3.84. Found: C, 83.65; H, 5.83; N, 6.72; O, 3.84; HRMS (ESI): $m / z=416.1889$ $\left([\mathrm{M}]^{+}\right)$, calcd for $\mathrm{C}_{29} \mathrm{H}_{24} \mathrm{~N}_{2} \mathrm{O}: 416.1889$.

1-Benzyl-2-(3',4'-dimethoxyphenyl)-4,5-diphenylimidazole (4d): Mp $163-165^{\circ} \mathrm{C}$ (EtOH); IR(KBr): $v 2835$ $(\mathrm{CH}), 1600(\mathrm{CN}) \mathrm{cm}^{-1} ;{ }^{1} \mathrm{H}$ NMR $\left(300 \mathrm{MHz}, \mathrm{CDCl}_{3}\right): \delta_{\mathrm{H}}$ ppm 7.64 - 6.89 (m, 18H, Ar), 5.10 (s, 2H, $\mathrm{CH}_{2}$ ), 3.89 (s, $\left.3 \mathrm{H}, \mathrm{OCH}_{3}\right), 3.69\left(\mathrm{~s}, 3 \mathrm{H}, \mathrm{OCH}_{3}\right) ;{ }^{13} \mathrm{C} \mathrm{NMR}(75 \mathrm{MHz}$, $\left.\mathrm{CDCl}_{3}\right): \delta$ ppm 149.63, 148.80, 137.99, 134.52, 131.10, 128.89 , 128.75, 128.16, 127.42, 126.90, 126.42, 125.96,
$121.65,112.24,110.99,55.96,55.69,48.28$.

1-Benzyl-2-(4'-chlorophenyl)-4,5-diphenylimidazole (4e): $\mathrm{Mp} 163-165^{\circ} \mathrm{C}(\mathrm{EtOH})$; IR( $\left.\mathrm{KBr}\right): v 2832(\mathrm{CH})$, $1598(\mathrm{CN}) \mathrm{cm}^{-1} ;{ }^{1} \mathrm{H}$ NMR $\left(300 \mathrm{MHz}, \mathrm{CDCl}_{3}\right): \delta_{\mathrm{H}} \mathrm{ppm}$ 8.00 - 6.82 (m, 19H, Ar), 5.09 (s, 2H, $\left.\mathrm{CH}_{2}\right) ;{ }^{13} \mathrm{C} \mathrm{NMR}$ $\left(75 \mathrm{MHz}, \mathrm{CDCl}_{3}\right): \delta$ ppm 146.92, 137.37, 135.06, 131.08, $130.31,129.98,129.11,128.92,128.79,128.20,126.85$, $125.92,48.34$.

1-Benzyl-2-(4'-ethylphenyl)-4,5-diphenylimidazole (4f): $\mathrm{Mp} 138-140^{\circ} \mathrm{C}(\mathrm{EtOH})$; IR(KBr): $v 2958(\mathrm{CH})$, 1597 (CN), 1492 (CC), 1446, $1388 \mathrm{~cm}^{-1} ;{ }^{1} \mathrm{H}$ NMR (300 $\left.\mathrm{MHz}, \mathrm{CDCl}_{3}\right): \delta_{\mathrm{H}}$ ppm $7.59-6.83(\mathrm{~m}, 19 \mathrm{H}, \mathrm{Ar}), 5.11(\mathrm{~s}$, $\left.2 \mathrm{H}, \mathrm{CH}_{2}\right), 2.67$ (q, 2H, $\left.J=6 \mathrm{~Hz}, \mathrm{CH}_{2}\right), 1.24(\mathrm{t}, 3 \mathrm{H}, J=6$ $\left.\mathrm{Hz}, \mathrm{CH}_{3}\right) ;{ }^{13} \mathrm{C}$ NMR $\left(75 \mathrm{MHz}, \mathrm{CDCl}_{3}\right): \delta$ ppm 148.20, $145.13,137.88,137.63,131.04,128.97,128.70,128.51$, $128.08,128.02,126.73,126.26,125.98,48.22,28.66$, 15.41. Anal. Calcd for $\mathrm{C}_{30} \mathrm{H}_{26} \mathrm{~N}_{2}$ : C, 86.92; H, 6.32; N, 6.76. Found: C, 86.90; H, 6.33; N, 6.78. HRMS (ESI): $m / z$ $=414.2098\left([\mathrm{M}]^{+}\right)$, calcd for $\mathrm{C}_{30} \mathrm{H}_{26} \mathrm{~N}_{2}$ : 414.2096. 1-Benzyl-2-(3'-bromophenyl)-4,5-diphenylimidazole (4g): $\mathrm{Mp}$ $148-150^{\circ} \mathrm{C}(\mathrm{EtOH}) ; \mathrm{IR}(\mathrm{KBr}): v 2949(\mathrm{CH}), 1601(\mathrm{CN})$ $\mathrm{cm}^{-1} ;{ }^{1} \mathrm{H}$ NMR $\left(300 \mathrm{MHz}, \mathrm{CDCl}_{3}\right): \delta_{\mathrm{H}} \mathrm{ppm} 7.66-6.82$ $(\mathrm{m}, 19 \mathrm{H}, \mathrm{Ar}), 5.11\left(\mathrm{~s}, 2 \mathrm{H}, \mathrm{CH}_{2}\right) ;{ }^{13} \mathrm{C} \mathrm{NMR}(75 \mathrm{MHz}$, $\left.\mathrm{CDCl}_{3}\right): \delta \mathrm{ppm} 132.17,131.86,130.99,130.00,129.04$, $128.86,128.77,128.69,128.57,128.11,127.51,127.22$, $126.75,126.51,125.98,125.90,48.31$.

1-Benzyl-2-(4'-nitrophenyl)-4,5-diphenylimidazole (4h): $\mathrm{Mp} 170-172^{\circ} \mathrm{C}$ (EtOH); IR(KBr): v 2963 (CH), $1608(\mathrm{CN}) \mathrm{cm}^{-1} ;{ }^{1} \mathrm{H}$ NMR $\left(300 \mathrm{MHz}, \mathrm{CDCl}_{3}\right): \delta_{\mathrm{H}} \mathrm{ppm}$ 8.12 - $6.73(\mathrm{~m}, 19 \mathrm{H}, \mathrm{Ar}), 4.88\left(\mathrm{~s}, 2 \mathrm{H}, \mathrm{CH}_{2}\right) ;{ }^{13} \mathrm{C} \mathrm{NMR}$ $\left(\mathrm{CDCl}_{3}\right): \delta$ ppm 148.92, 143.00, 136.57, 133.23, 131.10, $128.93,128.79,128.38,128.05,126.64,126.48,126.41$, $124.65,48.27$.

2-(2',5'-Dimethylphenyl)-1,4,5-triphenylimidazole (4i): Mp 173 - $175^{\circ} \mathrm{C}(\mathrm{EtOH}) ; \mathrm{IR}(\mathrm{KBr}): v 2942(\mathrm{CH}), 1598$ $(\mathrm{CN}), 1492(\mathrm{CC}) \mathrm{cm}^{-1}$; ${ }^{1} \mathrm{H}$ NMR $\left(300 \mathrm{MHz}, \mathrm{CDCl}_{3}\right): \delta_{\mathrm{H}}$ ppm $7.61-6.87$ (m, 18H, ArH), $2.23\left(\mathrm{~s}, 3 \mathrm{H}, \mathrm{CH}_{3}\right), 2.10$ $\left(\mathrm{s}, 3 \mathrm{H}, \mathrm{CH}_{3}\right) ;{ }^{13} \mathrm{C} \mathrm{NMR}\left(75 \mathrm{MHz}, \mathrm{CDCl}_{3}\right): \delta \mathrm{ppm} 147.59$, $137.64,136.50,134.74,134.64,134.51,131.79,130.94$, $130.78,130.40,129.83,129.73,129.07,128.46,128.37$, $128.05,127.78,127.71,127.49,127.41,126.44,20.73$, 19.67; Anal. Calcd for $\mathrm{C}_{29} \mathrm{H}_{24} \mathrm{~N}_{2}$ : C, 86.97; H, 6.04; N, 6.99. Found: C, 86.90; H, 6.04; N, 6.89. HRMS(ESI): $m / z=400.1939\left([\mathrm{M}]^{+}\right)$, calcd for $\mathrm{C}_{29} \mathrm{H}_{24} \mathrm{~N}_{2}: 400.1939$.

1-(4'-nitrophenyl)-2-(4'-chlorophenyl)-4,5-diphenylimidazole (4q): Mp $122-124^{\circ} \mathrm{C}(\mathrm{EtOH}) ; \mathrm{IR}(\mathrm{KBr}): v 2928$ (CH), $1590(\mathrm{CN}), 1489$ (CC), 1351 (C-NO2), 739 (CCl) $\mathrm{cm}^{-1} ;{ }^{1} \mathrm{H}$ NMR $\left(300 \mathrm{MHz}, \mathrm{CDCl}_{3}\right): \delta_{\mathrm{H}} \mathrm{ppm} 8.05-7.03$ $(18 \mathrm{H}, \mathrm{m}, \mathrm{ArH}) ;{ }^{13} \mathrm{C} \mathrm{NMR}\left(75 \mathrm{MHz}, \mathrm{CDCl}_{3}\right): \delta \mathrm{ppm}$ $194.66,152.50,134.94,132.87,129.88,129.02,128.60$, 127.77, 127.54, 126.47, 126.33, 113.31; Anal. Calcd for $\mathrm{C}_{27} \mathrm{H}_{18} \mathrm{~N}_{3} \mathrm{O}_{2} \mathrm{Cl}: \mathrm{C}, 71.76 ; \mathrm{H}, 4.01 ; \mathrm{N}, 9.30 ; \mathrm{O}, 7.08 ; \mathrm{Cl}$, 7.85. Found: C, 71.68; H, 4.04; N, 9.32; O, 7.09; Cl, 7.83. HRMS (ESI): $m / z=451.1089\left([\mathrm{M}]^{+}\right)$, calcd for 
$\mathrm{C}_{27} \mathrm{H}_{18} \mathrm{~N}_{3} \mathrm{O}_{2} \mathrm{Cl}: 451.1088$.

1-(4'-Nitrophenyl)-2-(3',4'-dimethoxyphenyl)-4,5-diphenylimidazole (4r): $\mathrm{Mp} 125-126^{\circ} \mathrm{C}(\mathrm{EtOH}) ; \mathrm{IR}(\mathrm{KBr})$ : v $2939(\mathrm{CH}), 1597(\mathrm{CN}), 1489(\mathrm{CC}), 1346(\mathrm{C}-\mathrm{NO} 2)$, $1246(\mathrm{CO}) \mathrm{cm}^{-1} ;{ }^{1} \mathrm{H}$ NMR $\left(300 \mathrm{MHz}, \mathrm{CDCl}_{3}\right): \delta_{\mathrm{H}} \mathrm{ppm}$ 8.14 - 8.11 (m, 2H, ArH), 7.59 - 7.56 (m, 2H, ArH), 7.30 - $7.12(\mathrm{~m}, 12 \mathrm{H}, \mathrm{ArH}), 6.70(\mathrm{~s}, 1 \mathrm{H}, \mathrm{ArH}), 3.86(\mathrm{~s}, 3 \mathrm{H}$, $\left.\mathrm{OCH}_{3}\right), 3.78$ (s, $\left.3 \mathrm{H}, \mathrm{OCH}_{3}\right) ;{ }^{13} \mathrm{C} \mathrm{NMR}\left(75 \mathrm{MHz}, \mathrm{CDCl}_{3}\right)$ : $\delta$ ppm 149.60, 133.72, 131.02, 129.90, 129.18, 128.78, 128.56, 128.24, 127.40, 126.99, 124.36, 121.91, 112.28, 110.63, 55.83; Anal. Calcd for $\mathrm{C}_{29} \mathrm{H}_{23} \mathrm{~N}_{3} \mathrm{O}_{4}$ : C, 72.94; $\mathrm{H}$, 4.85; N, 8.80; O, 13.40. Found: C, 72.96; H, 4.83; N, 8.82; O, 13.49. HRMS (ESI): $m / z=477.1689\left([\mathrm{M}]^{+}\right)$, calcd for $\mathrm{C}_{29} \mathrm{H}_{23} \mathrm{~N}_{3} \mathrm{O}_{4}: 477.1689$.

\subsection{Synthesis of Compounds 4(k-p)}

Carried as per the general procedure with $0.5 \mathrm{~mL}$ of aliphatic amines (methyl amine and ethyl amine).

1-methyl-2-(4'-chlororphenyl)-4,5-diphenylimidazole (4k): $\mathrm{Mp} 192-194^{\circ} \mathrm{C}(\mathrm{EtOH})$; IR(KBr): v $2925(\mathrm{CH})$, 1604 (CN), 1483 (CC), 735 (CCl) cm ${ }^{-1} ;{ }^{1} \mathrm{H}$ NMR (300 $\left.\mathrm{MHz}, \mathrm{CDCl}_{3}\right): \delta_{\mathrm{H}} \mathrm{ppm} 7.71-7.18(\mathrm{~m}, 14 \mathrm{H}, \mathrm{ArH}), 3.50(\mathrm{~s}$, $\left.3 \mathrm{H}, \mathrm{NCH}_{3}\right) ;{ }^{13} \mathrm{C}$ NMR $\left(75 \mathrm{MHz}, \mathrm{CDCl}_{3}\right): \delta$ ppm 146.79 , $137.98,134.90,134.44,130.97,130.89,130.84,130.32$, $129.38,129.15,129.05,128.92,128.77,128.20,127.01$, 126.54, 33.29; Anal. Calcd for $\mathrm{C}_{22} \mathrm{H}_{17} \mathrm{~N}_{2} \mathrm{Cl}$ : C, 76.63; H, 4.97; N, 8.12; Cl, 10.28. Found: C, 76.65; H, 4.99; N, 8.19; Cl, 10.31; HRMS(ESI): $m / z=344.1082\left([\mathrm{M}]^{+}\right)$, calcd for $\mathrm{C}_{22} \mathrm{H}_{17} \mathrm{~N}_{2} \mathrm{Cl}$ : 344.1080 .

1-methyl-2-(4'-ethylphenyl)-4, 5-diphenylimidazole (4I): $\mathrm{Mp} 127$ - 130 ${ }^{\circ} \mathrm{C}$ (EtOH); IR(KBr): v $2920(\mathrm{CH})$, $1597(\mathrm{CN}), 1480(\mathrm{CC}) \mathrm{cm}^{-1} ;{ }^{1} \mathrm{H} \mathrm{NMR}\left(300 \mathrm{MHz}, \mathrm{CDCl}_{3}\right)$ : $\delta_{\mathrm{H}} \mathrm{ppm} 7.67-7.19(\mathrm{~m}, 14 \mathrm{H}, \mathrm{ArH}), 3.51\left(\mathrm{~s}, 3 \mathrm{H}, \mathrm{NCH}_{3}\right)$, 2.73 (q, $2 \mathrm{H}, J=6 \mathrm{~Hz}, \mathrm{CH}_{2}$ ), 1.29 (t, $3 \mathrm{H}, J=6 \mathrm{~Hz}, \mathrm{CH}_{3}$ ); ${ }^{13} \mathrm{C}$ NMR $\left(75 \mathrm{MHz}, \mathrm{CDCl}_{3}\right): \delta$ ppm 134.84, 130.79, $129.84,128.95,128.43,128.00,127.97,126.87,33.06$, 28.65, 15.40; Anal. Calcd for $\mathrm{C}_{24} \mathrm{H}_{22} \mathrm{~N}_{2}$ : C, 85.17; H, 6.55 ; N, 8.28. Found: C, 85.19; H, 6.58; N, 8.29. HRMS(ESI): $m / z=338.1786\left([\mathrm{M}]^{+}\right.$), calcd for $\mathrm{C}_{24} \mathrm{H}_{22} \mathrm{~N}_{2}$ : 338.1783 .

1-Ethyl-2-(2'-methoxyphenyl)-4,5-diphenylimidazole (4m):Mp $123-125^{\circ} \mathrm{C}(\mathrm{EtOH})$; IR( $\left.\mathrm{KBr}\right): v 2924(\mathrm{CH})$, 1600 (CN), 1462 (CC), 1260 (CO) cm ${ }^{-1} ;{ }^{1} \mathrm{H}$ NMR (300 $\left.\mathrm{MHz}, \mathrm{CDCl}_{3}\right): \delta_{\mathrm{H}}$ ppm $7.57-7.09(14 \mathrm{H}, \mathrm{m}, \mathrm{ArH}), 3.85$ $\left(3 \mathrm{H}, \mathrm{s}, \mathrm{OCH}_{3}\right), 3.77\left(2 \mathrm{H}, \mathrm{q}, J=6 \mathrm{~Hz}, \mathrm{CH}_{2}\right), 0.90(3 \mathrm{H}, \mathrm{t}$, $\left.J=6 \mathrm{~Hz}, \mathrm{CH}_{3}\right) ;{ }^{13} \mathrm{C}$ NMR $\left(75 \mathrm{MHz}, \mathrm{CDCl}_{3}\right): \delta \mathrm{ppm}$ $157.53,144.69,137.57,134.76,132.66,131.77,130.92$, $130.81,128.93,128.68,128.40,127.88,126.72,125.91$, 120.88, 120.66, 110.85, 55.47, 39.47, 15.85; Anal. Calcd for $\mathrm{C}_{24} \mathrm{H}_{22} \mathrm{~N}_{2} \mathrm{O}$ : C, 81.33; H, 6.26; N, 7.90; O, 4.51. Found: C, 81.41; H, 6.28; N, 7.95; O, 4.56. HRMS(ESI): $m / z=354.1736\left([\mathrm{M}]^{+}\right)$, calcd for $\mathrm{C}_{24} \mathrm{H}_{22} \mathrm{~N}_{2} \mathrm{O}: 354.1732$.

1-Ethyl-2(4'-chlorophenyl)-4,5-diphenylimidazole (4n): $\mathrm{Mp} 311-313^{\circ} \mathrm{C}(\mathrm{EtOH}) ; \mathrm{IR}(\mathrm{KBr}): v 2930(\mathrm{CH})$,
$1596(\mathrm{CN}) \mathrm{cm}^{-1} ;{ }^{1} \mathrm{H}$ NMR $\left(300 \mathrm{MHz}, \mathrm{CDCl}_{3}\right): \delta_{\mathrm{H}} \mathrm{ppm}$ 7.68 - $7.16(14 \mathrm{H}, \mathrm{m}, \mathrm{ArH}), 3.94\left(2 \mathrm{H}, \mathrm{q}, J=6 \mathrm{~Hz}, \mathrm{CH}_{2}\right)$, $1.03\left(3 \mathrm{H}, \mathrm{t}, J=6 \mathrm{~Hz}, \mathrm{CH}_{3}\right) ;{ }^{13} \mathrm{C} \mathrm{NMR}\left(75 \mathrm{MHz}, \mathrm{CDCl}_{3}\right)$ : $\delta$ ppm 194.57, 146.04, 137.95, 134.90, 134.33, 132.91, $131.26,130.98,130.34,129.89,129.82,129.66,129.08$, $129.01,128.87,128.76,128.05,126.70,126.30,39.65$, 16.22 .

1-Ethyl-2-(3',4'-dimethoxyphenyl)-4,5-diphenylimidazole (4o): $\mathrm{Mp} 173-175^{\circ} \mathrm{C}$ (EtOH); $\mathrm{IR}(\mathrm{KBr}): v 2928$ (CH), $1608(\mathrm{CN}), 1482(\mathrm{CC}), 1258(\mathrm{CO}) \mathrm{cm}^{-1} ;{ }^{1} \mathrm{H} \mathrm{NMR}$ $\left(300 \mathrm{MHz}, \mathrm{CDCl}_{3}\right): \delta_{\mathrm{H}} \mathrm{ppm} 7.54-6.98(13 \mathrm{H}, \mathrm{m}, \mathrm{ArH})$, $3.96-3.90\left(8 \mathrm{H}, \mathrm{m}, 2 \mathrm{OCH}_{3}\right.$ and $\left.\mathrm{CH}_{2}\right), 1.02(3 \mathrm{H}, \mathrm{t}, J=6$ $\left.\mathrm{Hz}, \mathrm{CH}_{3}\right) ;{ }^{13} \mathrm{C}$ NMR $\left(75 \mathrm{MHz}, \mathrm{CDCl}_{3}\right): \delta \mathrm{ppm} \mathrm{149.64,}$ $149.04,137.54,134.63,131.66,131.08,129.21,129.08$, $128.66,128.04,126.77,126.18,124.11,121.51,112.72$, $110.98,56.04,55.99,39.65,16.28$; Anal. Calcd for $\mathrm{C}_{25} \mathrm{H}_{24} \mathrm{~N}_{2} \mathrm{O}_{2}$ : C, 78.10; H, 6.29; N, 7.29; O, 8.32. Found: C, 78.14; H, 6.27; N, 7.32; O, 8.35. HRMS (ESI): $\mathrm{m} / \mathrm{z}=$ $384.1839\left([\mathrm{M}]^{+}\right)$, calcd for $\mathrm{C}_{24} \mathrm{H}_{22} \mathrm{~N}_{2} \mathrm{O}: 384.1838$.

1-Ethyl-2-(2',6'-dichlorophenyl)-4,5-di(4'-methylphenyl)imidazole $(4 p)$ : $\mathrm{Mp} 135-137^{\circ} \mathrm{C}(\mathrm{EtOH}) ; \mathrm{IR}(\mathrm{KBr})$ : v $2924(\mathrm{CH}), 1605(\mathrm{CN}), 1450(\mathrm{CC}), 738(\mathrm{CCl}) \mathrm{cm}^{-1} ;{ }^{1} \mathrm{H}$ NMR $\left(300 \mathrm{MHz}, \mathrm{CDCl}_{3}\right): \delta_{\mathrm{H}} \mathrm{ppm} 7.46-7.00(11 \mathrm{H}, \mathrm{m}$, $\operatorname{ArH}), 3.66\left(2 \mathrm{H}, \mathrm{q}, J=6 \mathrm{~Hz}, \mathrm{NCH}_{2}\right), 2.45\left(3 \mathrm{H}, \mathrm{s}, \mathrm{CH}_{3}\right)$, $2.28\left(3 \mathrm{H}, \mathrm{s}, \mathrm{CH}_{3}\right), 1.00\left(3 \mathrm{H}, \mathrm{t}, J=6 \mathrm{~Hz}, \mathrm{CH}_{3}\right) ;{ }^{13} \mathrm{C} \mathrm{NMR}$

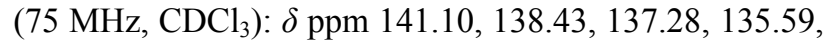
$131.78,131.22,130.86,129.72,128.65,128.22,128.08$, 126.54, 39.32, 21.40, 21.10, 15.88; Anal. Calcd for $\mathrm{C}_{25} \mathrm{H}_{22} \mathrm{~N}_{2} \mathrm{Cl}_{2}$ : C, 71.26; H, 5.26; N, 6.65; Cl, 16.83 . Found: $\mathrm{C}, 71.29 ; \mathrm{H}, 5.32 ; \mathrm{N}, 6.64 ; \mathrm{Cl}, 16.84$. HRMS (ESI): $m / z=420.1160\left([\mathrm{M}]^{+}\right)$, calcd for $\mathrm{C}_{25} \mathrm{H}_{22} \mathrm{~N}_{2} \mathrm{Cl}_{2}$ : 420.1160 .

\section{Acknowledgements}

J. D. is grateful to CSIR, New Delhi for award of SRF. The authors are also grateful to SAIF, Gauhati University for recording the single crystal XRD data and Mr S.J. Bharali for analyzing the data.

\section{REFERENCES}

[1] M. R. Grimmet and A. R. Katritzky, "In Comprehensive Heterocyclic Chemistry," In: C. W. Rees, Ed., Pergamon Press, London, Vol. 5, 1984, p. 374.

[2] D. Norwood, E. Branch, B. Smith and M. Honeywell, "Olmesartan Medoxomil for Hypertension: A Clinical Review," Drug Forecast, Vol. 27, No. 12, 2002, pp. 611618.

[3] S. L. Abrahams, R. J. Hazen, A. G. Batson and A. P. Phillips, "Trifenagrel: A Chemically Novel Platelet Aggregation Inhibitor," Journal of Pharmacology and Experimental Therapeutics, Vol. 249, No. 2, 1989, pp. 359365.

[4] S. E. Wolkenberg, D. D. Wisnoski, W. H. Leister, Y. Wang, Z. Zhao and C. W. Lindsley, "Efficient Synthesis 
of Imidazoles from Aldehydes and 1,2-Diketones Using Microwave Irrediation," Organic Letters, Vol. 6, No. 9, 2004, pp. 1453-1456. http://dx.doi.org/10.1021/o1049682b

[5] J. G. Lombardino and E. H. Wiseman, "Preparation and Antiinflammatory Activity of Some Nonacidic Trisubstituted Imidazoles," Journal of Medicinal Chemistry, Vol. 17, No. 11, 1974, pp. 1182-1188. http://dx.doi.org/10.1021/jm00257a011

[6] J. G. Lombardino, "Pharmaceutical Imidazoles," Ger. Offen, DE 2155558 A 19720629, 1972.

[7] A. P. Phillips, H. L. White and S. Rosen, "Antithrombotic Triphenylimidazoles," European Patent, EP 58890 A1 19820901, 1982.

[8] J. Zhu, "Recent Developments in the Isonitrile-Based Multicomponent Synthesis of Heterocycles," European Journal of Organic Chemistry, Vol. 2003, No. 7, 2003, pp. 1133-1144. http://dx.doi.org/10.1002/ejoc.200390167

[9] R. V. A. Orru and M. de Greef, "Recent Advances in Solution-Phase Multicomponent Methodology for the Synthesis of Heterocyclic Compounds," Synthesis, Vol. 2003, No. 10, 2003, pp. 1471-1499. http://dx.doi.org/10.1055/s-2003-40507

[10] D. J. Ramon and M. Yus, "Asymmetric Multicomponent Reactions (AMCRs): The New Frontier," Angewandte Chemie International Edition, Vol. 44, No. 11, 2005, pp. 1602-1634. http://dx.doi.org/10.1002/anie.200460548

[11] D. E. Frantz, L. Morency, A. Soheili, J. A. Murry, E. J. J. Grabowski and R. D. Tillyer, "Synthesis of Substituted Imidazole via Organocatalysis," Organic Letters, Vol. 6, No. 5, 2004, pp. 843-846. http://dx.doi.org/10.1021/ol0498803

[12] C. Zhang, E. J. Moran, T. F. Woiwade, K. M. Short and A. M. M. Mjalli, "Synthesis of Tetrasubstituted Imidazoles via $\alpha$-(N-acyl-N-alkylamino)- $\beta$-ketoamides on Wang Resin," Tetrahedron Letters, Vol. 37, No. 6, 1996, pp. 751754. http://dx.doi.org/10.1016/0040-4039(95)02310-0

[13] I. Lantos, W-Y. Zhang, X. Shui and D. S. Eggleston, "Synthesis of Imidazole via Hetero-Cope Rearrange ment," The Journal of Organic Chemistry, Vol. 58, No. 25, 1993, pp. 7092-7095. http://dx.doi.org/10.1021/jo00077a033

[14] T. J. Donohoe, M. A. Kabeshov, A. H. Rathi and I. E. D. Smith, "Direct Preparation of Thiazoles, Imidazoles, Imidazopyridines and Thiazolidines from Alkenes," Organic \& Biomolecular Chemistry, Vol. 10, No. 5, 2012, pp. 1093-1101. http://dx.doi.org/10.1039/c1ob06587d

[15] B. Hu, Z. Wang, N. Ai, J. Zheng, X-H. Liu, S. Shan and Z. Wang, "Catalyst-Free Preparation of 1,2,4,5-Tetrasubstituted Imidazoles from a Novel Unexpected Domino Reaction of 2-Azido Acrylates and Nitrones," Organic Letters, Vol. 13 , No. 24, 2011, pp. 6362-6365. http://dx.doi.org/10.1021/ol202650z

[16] B. H. Lipshutz and M. C. Morey, "An Approach to the Cyclopeptide Alkaloids (Phencyclopeptines) via Heterocyclic Diamide/Dipeptide Equivalents. Preparation and N-Alkylation Studies of 2,4(5)-Disubstituted Imidazoles," The Journal of Organic Chemistry, Vol. 48 , No. 21, 1983, pp. 3745-3750. http://dx.doi.org/10.1021/jo00169a027
[17] B. Sadeghi, B. B. F. Mirjalili and M. M. Hashemi, " $\mathrm{BF}_{3} \cdot \mathrm{SiO}_{2}$ : An Efficient Reagent System for the One-Pot Synthesis of 1,2,4,5-Tetrasubstituted Imidazoles," Tetrahedron Letters, Vol. 49, No. 16, 2008, pp. 2575-2577. http://dx.doi.org/10.1016/j.tetlet.2008.02.100

[18] R. Consonni, P. D. Croce, R. Ferraccioli and C. L. Rosa, "A New Approach to Imidazole Derivatives," Journal of Chemical Research (Synopses), No. 7, 1991, pp. 188-189.

[19] D. A. Evans and K. M. Lundy, "Synthesis of Diphthamide: The Target of Diphtheria Toxin Catalyzed ADP-Ribosylation in Protein Synthesis Elongation Factor 2," Journal of the American Chemical Society, Vol. 114, No. 4, 1992, pp. 1495-1496.

http://dx.doi.org/10.1021/ja00030a063

[20] P. Schneiders, J. Heinze and H. Baumgartel, "Synthesis of 4,4',5,5'-Tetrasubstituted Di-2-imidazolyl Derivatives, Starting Materials for the Synthesis of 1,4,5,8-Tetraazafulvalenes," Chemische Berichte, Vol. 106, No. 7, 1973, pp. 2415-2417. http://dx.doi.org/10.1002/cber.19731060739

[21] C. F. Claiborne, N. J. Liverton and K. T. Nguyen, “An Efficient Synthesis of Tetrasubstituted Imidazole from N-(2-Oxo)-Amides," Tetrahedron Letters, Vol. 39, No. 49, 1998, pp. 8939-8942. http://dx.doi.org/10.1016/S0040-4039(98)02058-9

[22] S. Narayana Murthy, B. Madhav and Y. V. D. Nageswar, "DABCO as a Mild and Efficient Catalytic System for the Synthesis of Highly Substituted Imidazoles via MultiComponent Condensation Strategy," Tetrahedron Letters, Vol. 51, No. 40, 2010, pp. 5252-5257. http://dx.doi.org/10.1016/j.tetlet.2010.07.128

[23] S. Kantevari, S. V. N. Vuppalapati, D. O. Biradar and L. Nagarapu, "Highly Efficient, One-Pot, Solvent-Free Synthesis of Tetrasubstituted Imidazoles Using $\mathrm{HClO}_{4}-\mathrm{SiO}_{2}$ as Novel Heterogeneous Catalyst," Journal of Molecular Catalysis A: Chemical, Vol. 266, No. 1-2, 2007, pp. 109113. http://dx.doi.org/10.1016/j.molcata.2006.10.048

[24] M. Kidwai and P. Mothsra, "A One-Pot Synthesis of 1,2, 4,5-Tetraarylimidazole Using Molecular Iodine as an Efficient Catalyst," Tetrahedron Letters, Vol. 47, No. 29, 2006, pp. 5029-5031. http://dx.doi.org/10.1016/j.tetlet.2006.05.097

[25] S. Balalaie, M. M. Hashemi and M. Akhbari, "A Novel One-Pot Synthesis of Tetrasubstituted Imidazoles under Solvent-Free Conditions and Microwave Irradiation," Tetrahedron Letters, Vol. 44, No. 8, 2003, pp. 1709-1711. http://dx.doi.org/10.1016/S0040-4039(03)00018-2

[26] S. Samai, G. C. Nandi, P. Singh and M. S. Singh, "LProline: An Efficient Catalyst for the One-Pot Synthesis of 2,4,5-Trisubstituted and 1,2,4,5-Tetrasubstituted Imidazoles," Tetrahedron, Vol. 65, No. 49, 2009, pp. 10155 10161. http://dx.doi.org/10.1016/j.tet.2009.10.019

[27] S. Balalaie and A. Arabanian, "One-Pot Synthesis of Tetrasubstituted Imidazoles Catalyzed by Zeolite HY and Silica Gel under Microwave Irradiation," Green Chemistry, Vol. 2, No. 6, 2000, pp. 274-276. http://dx.doi.org/10.1039/b006201o

[28] M. M. Heravi, F. Derikvand and F. F. Bamoharramb, "Highly Efficient, Four-Component One-Pot Synthesis of 
Tetrasubstituted Imidazoles Using Keggin-Type Heteropolyacids as Green and Reusable Catalysts," Journal of Molecular Catalysis A: Chemical, Vol. 263, No. 1-2, 2007, pp. 112-114.

http://dx.doi.org/10.1016/j.molcata.2006.08.048

[29] M. R. Manafi, P. Manafi and M. R. Kalaee, "Versatile Microwave-Assisted and Lanthanum Chloride Catalysed Synthesis of Polysubstituted Imidazoles Using Urea/ Thiourea as Benign Source of Ammonia," E-Journal of Chemistry, Vol. 9, No. 4, 2012, pp. 1773-1777. http://dx.doi.org/10.1155/2012/396127

[30] H. R. Shaterian and M. Ranjbar, "An Environmental Friendly Approach for the Synthesis of Highly Substituted Imidazoles Using Brønsted Acidic Ionic Liquid, N-Methyl-2-Pyrrolidonium Hydrogen Sulphate, as Reusable Catalyst," Journal of Molecular Liquids, Vol. 160, No. 1, 2011, pp. 40-49. http://dx.doi.org/10.1016/j.molliq.2011.02.012

[31] P. Wasserscheid and T. Welton, "Ionic Liquids in Synthesis," 2nd Edition, Wiley-VCH, Weinheim, 2007. http://dx.doi.org/10.1002/9783527621194
[32] Y. Izumi, K. Urabe and M. Onaka, "Zeolite, Clay and Heteropolyacid in Organic Reactions," Kodansha/VCH, Tokyo, 1992.

[33] T. Okuhara, N. Mizuno and M. Misono, "Catalytic Che mistry of Heteropoly Compounds," Advances in Catalysis, Vol. 41, 1996, pp. 113-252. http://dx.doi.org/10.1016/S0360-0564(08)60041-3

[34] P. J. Das and J. Das, "Synthesis of Aryl/alkyl(2,2'-bis-3methylindolyl)methanes and Aryl(3,3'-bisindolyl)Methanes Promoted by Secondary Amine Based Ionic Liquids and Microwave Irradiation," Tetrahedron Letters, Vol. 53, No. 35, 2012, pp. 4718-4720. http://dx.doi.org/10.1016/j.tetlet.2012.06.106

[35] I. V. Kozhevnikov, "Catalysis by Heteropoly Acids and Multicomponent Polyoxometalates in Liquid-Phase Reactions," Chemical Review, Vol. 98, No. 1, 1998, pp. 171198. http://dx.doi.org/10.1021/cr960400y 